\title{
Some evidence that one-to-one dietary interventions in the dental setting can change behaviour
}

\author{
Abstracted from \\ Harris R, Gamboa A, Dailey Y, Ashcroft A. \\ One-to-one dietary interventions undertaken in a dental setting to change dietary behaviour. \\ Cochrane Database of Syst Rev. 2012; Issue 3. Art. No. CD006540 \\ Address for Correspondence Luisa Fernandez Mauleffinch, Review Group Co-ordinator, \\ Cochrane Oral Health Group, MANDEC, School of Dentistry, University of Manchester, \\ Higher Cambridge Street, Manchester, M15 6FH, UK. E-mail luisa.fernandez@manchester.ac.uk
}

\section{Question: Are one-to-one dietary interventions in a dental care setting effective in changing behaviour?}

Data sources The Cochrane Oral Health Group Trials Register CENTRAL, Medline, Embase, PsycINFO, CINAHL, Web of Science conference proceedings (IADR and ORCA), reference lists and Dissertations Abstracts were searched.

Study selection Randomised controlled trials assessing the effectiveness of 1:1 dietary interventions in a dental care setting were included. This could be brief advice, skills training, self help materials, counselling or lifestyle strategies delivered by any member of the dental team.

Data extraction and synthesis Two reviewers independently screened and abstracted data with disagreements resolved by discussion and a third review author. The Cochrane risk of bias assessment tool was used. Results Five studies were included; two were at high risk of bias, three were at unclear risk of bias. Two were multi-intervention studies where the dietary intervention was one component of a wider programme of prevention, but where data on dietary behaviour change were reported. One of the single intervention studies concerned caries prevention. The others concerned general health outcomes. No studies were aimed at preventing tooth erosion. Four out of five studies found a significant change in dietary behaviour in at least one of the primary outcomes. Conclusions There is some evidence that one-to-one dietary interventions in the dental setting can change behaviour, although the evidence is greater for interventions aiming to change fruit/vegetable and alcohol consumption than for those aiming to change dietary sugar consumption. There is a need for more studies, particularly in the dental practice setting, as well as greater methodological rigour in the design, statistical analysis and reporting of such studies.

\section{Commentary}

Preventing caries through dietary control remains the Holy Grail in oral health improvement. Undoubtedly, fluoride and fissure sealing interventions (which are usually part of caries preventive programmes delivered at the chairside) can significantly reduce caries

This paper is based on a Cochrane Review published in the Cochrane Library 2012, issue 3 (see www.thecochranelibrary.com for information). Cochrane Reviews are regularly updated as new evidence emerges and in response to feedback, and the Cochrane Library should be consulted for the most recent version of the review. experience. However, dental decay, one of the commonest diseases in the world, could be almost eradicated by removing from the diet a foodstuff that is not essential for general nutrition. The problem for health care professionals, though, is that encouraging behaviour change in their patients is notoriously difficult. Helping people to start doing something which would have health benefits for them is hard enough; getting them to stop doing something they are already doing, and which is associated with enjoyment, has seemed almost impossible. Anecdotally, many clinicians appear to have almost given up on dietary advice for their patients and it is rare to see behaviour change interventions as part of preventive programmes.

This important review shows that, in fact, simple one-to-one brief interventions, carried out in a dental setting, can bring about dietary behaviour change. One proviso is that the studies reported in this review are based on self-reported data. However, whilst this is an obvious limitation of these studies, it is difficult to see how else dietary changes could be assessed. Just three of the five studies related to changing sugar consumption to reduce dental caries, and more surprisingly, none related to dental erosion.

Only five studies (842 participants), met this review's strict inclusion criteria. This compares unfavourably with the number of studies included in similar reviews of other preventive interventions. For example, the Cochrane toothbrushing review ${ }^{1}$ included 74 studies and 42,300 children. Carrying out high quality research into dietary change presents many challenges, and there are other, perhaps more attractive, avenues for the researcher with time and budgetary constraints to explore. However, poor nutrition remains not just an oral health problem, but a general health problem, with significant long term health implications, including increased risk of coronary artery disease and diabetes. A recent review in England reported that around 30\% of children aged 2-15 years were overweight or obese (http://www.ic.nhs.uk/ webfiles/publications/003_Health_Lifestyles/OPAD12/Statistics_on_ Obesity_Physical_Activity_and_Diet_England_2012.pdf).

Oral health care professionals are ideally placed to help their patients develop good dietary habits, for the benefit of their oral, and general, health. This review, while indicating the need for more high quality clinical trials, shows that such interventions can be effective, and should be part of any chairside caries preventive programme.

Dafydd Evans

Dental School, University of Dundee, Dundee, Scotland, UK

1. Marinho VCC, Higgins JPT, Logan S, Sheiham A. Fluoride toothpastes for preventing dental caries in children and adolescents. Cochrane Database of Syst Rev. 2003; Issue 1. Art. No. CD002278. DOI: 10.1002/14651858.CD002278.

Evidence-Based Dentistry (2012) 13, 42. doi:10.1038/sj.ebd.6400855 